07,01

\title{
Динамическая модель упруго-пластического нормального столкновения сферических частиц при нелокальной пластичности
}

\author{
(С) Я.А. Ляшенко ${ }^{1,2}$, В.Л. Попов ${ }^{1,3,4, \text { ฯ }}$ \\ ${ }^{1}$ Берлинский технический университет, \\ Берлин, Германия \\ ${ }^{2}$ Сумский государственный университет, \\ Сумы, Украина \\ ${ }^{3}$ Национальный исследовательский Томский государственный университет, \\ Томск, Россия \\ ${ }^{4}$ Национальный исследовательский Томский политехнический университет, \\ Томск, Россия \\ TE-mail: v.popov@tu-berlin.de
}

(Поступила в Редакцию 10 июля 2017 г.)

Рассмотрена задача нормального столкновения сферической частицы с полупространством. Проведен учет нелокальной пластической деформации, в случае, когда предел прочности зависит от радиуса контакта, а также эффекта упрочнения деформируемого материала. Численно рассчитана величина безразмерного коэффициента восстановления нормальной скорости, как функция начальной скорости сферической частицы. Полученные данные хорошо совпадают с имеющимися в литературе экспериментальными результатами.

Работа выполнена при финансовой поддержке Немецкого научно-исследовательского сообщества (DFG), Министерства образования Российской Федерации и программы повышения конкурентоспособности Томского государственного университета. Я.А. Ляшенко выражает благодарность МОНУ за частичную финансовую поддержку в рамках проекта № 0116U006818.

DOI: $10.21883 /$ FTT.2018.03.45562.229

\section{1. Введение}

Одной из актуальных задач контактной механики является точное описание процесса соударения твердых тел. Элементарные акты соударения во многом определяют поведение гранулированных сред [1-3]. Изучение динамики гранулированных сред имеет практическое значение как для оптимизации технологических процессов (например, сушка и фасовка удобрений), так и при моделировании некоторых природных явлений, таких как землетрясения, перемещения ледников и оползни. Взаимодействие между контактирующими частицами изучается также с целью описания формирования и эволюции планетарных систем. К примеру, в работе [4], основываясь на данных, полученных космическим кораблем „Вояджер“, изучается эволюция колец Сатурна с точки зрения столкновений микро-астероидов.

Однако, несмотря на кажущуюся простоту описания процесса столкновения сферических частиц, этот вопрос до настоящего времени далек от полного понимания. Ситуация усложняется еще и тем, что существенное влияние на процесс столкновения могут оказывать адгезионные силы [5,6], шероховатость поверхностей, пластические деформации $[7,8]$, либо наличие третьего тела в зоне контакта (смазка, продукты износа). К примеру, при столкновении ледяных частиц они могут частично плавиться в зоне контакта, и при определенных условиях после столкновения частицы будут представлять единое целое. Подобная ситуация может наблюдаться и при наличии сил адгезии между контактирующими поверхностями - если начальная скорость частиц меньше определенного значения, за счет работы адгезионных сил происходит диссипация механической энергии, и частицы слипаются. На сегодняшний день существует большое количество теорий и экспериментов по столкновению твердых частиц. К примеру, в работе [8] предложена теория описания столкновений частиц с учетом пластических деформаций. Однако сам механизм пластических деформаций может быть различным, поэтому указанная теория описывает лишь часть возможных сценариев.

Одним из перспективных и развивающихся методов исследования механики столкновений является метод редукции размерности (MDR) [9]. К примеру, в рамках метода MDR было исследовано столкновение частиц с бесконечным коэффициентом трения в зоне контакта (без учета скольжения) [10], с конечным коэффициентом трения [11], а также с наличием сил адгезии между контактирующими телами [12]. Причем в указанных случаях численно была найдена функция, которая задает кинетические параметры после удара (нормальную и тангенциальную компоненту скоростей и циклическую скорость вращения сферического тела) как функцию соответствующих параметров до удара. Указанная функция записана в безразмерных параметрах, которые содержат материальные и геометрические характеристики соударяющихся сфер, что позволяет использовать полученные результаты для описания систем с различны- 
ми параметрами. Однако в стороне остался вопрос о влиянии пластических деформаций на процесс столкновения. В недавней работе [13] предложен критерий пластичности, и описаны особенности его применения в рамках метода MDR. Предлагаемая работа предпринята с целью описания упруго-пластического столкновения сферических тел и в особенности посвящена изучению влияния нелокальных пластических деформаций.

\section{2. Формулировка модели нормальных столкновений в рамках метода MDR}

В рамках метода редукции размерности для описания контакта аксиально симметричного упругого тела с упругим полупространством необходимо, во-первых, заменить оригинальный трехмерный профиль контактирующего с полупространством тела $z=f(r)$ на его одномерный эквивалент, который задается преобразованием Абеля [9]

$$
g(x)=|x| \int_{0}^{|x|} \frac{f^{\prime}(r)}{\sqrt{x^{2}-r^{2}}} d r .
$$

Для сферического индентора $f(r)=r^{2} / 2 R$ преобразование (1) приводит к зависимости $g(x)=x^{2} / R$. Взаимодействующие тела одновременно заменяются на упругое основание - последовательность невзаимодействующих пружин, имеющих нормальную жесткость

$$
k_{z}=E^{*} \Delta x,
$$

где $\Delta x-$ величина дискретизации (расстояние между пружинами), а

$$
\frac{1}{E^{*}}=\frac{1-v_{1}^{2}}{E_{1}}+\frac{1-v_{2}^{2}}{E_{2}}
$$

где $E_{1}-$ модуль упругости, $v_{1}-$ коэффициент Пуассона индентора, а $E_{2}$ и $v_{2}-$ соответствующие параметры упругого полупространства.

Третьим шагом в процедуре MDR является вдавливание индентора в упругое основание на глубину $d$, в результате чего индивидуальные пружины испытывают смещения

$$
u_{z}(x)=d-g(x)=d-\frac{x^{2}}{R} .
$$

Радиус контакта $a$ определяется условием $u_{z}(a)=0$, откуда $a=\sqrt{R d}$. Зная глубину индентирования $d$, можно определить действующую нормальную силу $F_{n}$. Для этого необходимо сложить силы натяжения от каждой пружины в контакте

$$
\begin{aligned}
F_{z}(a) & =E^{*} \int_{-a}^{a} u_{z}(x) d x \\
& =2 E^{*} \int_{0}^{\sqrt{R d}}\left(d-\frac{x^{2}}{R}\right) d x=\frac{4 E^{*} \sqrt{R d^{3}}}{3} .
\end{aligned}
$$

Отметим, что при численном моделировании необходимо пользоваться дискретным представлением интеграла (5), т. е. использовать эквивалентную сумму

$$
F_{z}=E^{*} \Delta x \sum_{\text {cont }} u_{z}\left(x_{i}\right),
$$

где производится суммирование по всему контакту. Функция $u_{z}\left(x_{i}\right)$, как и ранее, задается выражением (4).

Поскольку целью настоящей работы является описание упруго-пластического столкновения, мы должны ввести в MDR критерий пластичности. Такой критерий был предложен в работе [13]. Причем в [13] рассматривается специфический случай нелокальной пластичности, когда теряет смысл критическое напряжение текучести материала. Такая ситуация наблюдается для малых радиусов контакта. Нелокальность критерия пластичности ведет к зависимости „твердости“ от размера контакта. Насколько нам известно, применимо к механике столкновений ранее указанный критерий нелокальной пластичности не рассматривался. Согласно [13] действующая в контакте нормальная сила может быть записана в виде

$$
F_{z}=\pi q_{c} a,
$$

где $a-$ радиус контакта, а $q_{c}$ имеет смысл критической линейной плотности силы, при превышении которой начинается пластическая деформация. Критическая сила $f_{c}$, действующая на одну пружину, может быть найдена как

$$
f_{c}=\frac{F_{z}}{2 a / \Delta x} \text {. }
$$

Из (7) и (8) следует выражение для определения величины $f_{c}[13]$

$$
f_{c}=\frac{\pi}{2} q_{c} \Delta x,
$$

где $f_{c}$ - критическая (максимальная) упругая сила, при превышении которой начинается пластическое деформирование пружины. Однако критерий (9) пока не учитывает упрочнение материала, который подвергается пластической деформации.

Рассмотрим подробно процесс пластического деформирования выделенной пружины с учетом упрочнения. На рис. 1 показана известная схема нагружения индивидуальной пружины [14]. Здесь до критического значения $f_{c}(9)$ пружина деформируется упруго. Это упругий участок зависимости, на котором нормальная сила задается законом Гука

$$
F_{z}\left(x_{i}\right)=\left[u_{z}\left(x_{i}\right)-u_{z}^{p l}\left(x_{i}\right)\right] E^{*} \Delta x,
$$

в котором для рассматриваемого упругого участка $u_{z}^{p l}\left(x_{i}\right)=0$. На упругом участке при разгружении пружины она полностью восстанавливает свою первоначальную форму. Если же натяжение пружины превысит критическое значение

$$
u_{z}\left(x_{i}\right)>u_{z}^{\mathrm{crit}}=\frac{f_{c}}{E^{*} \Delta x},
$$




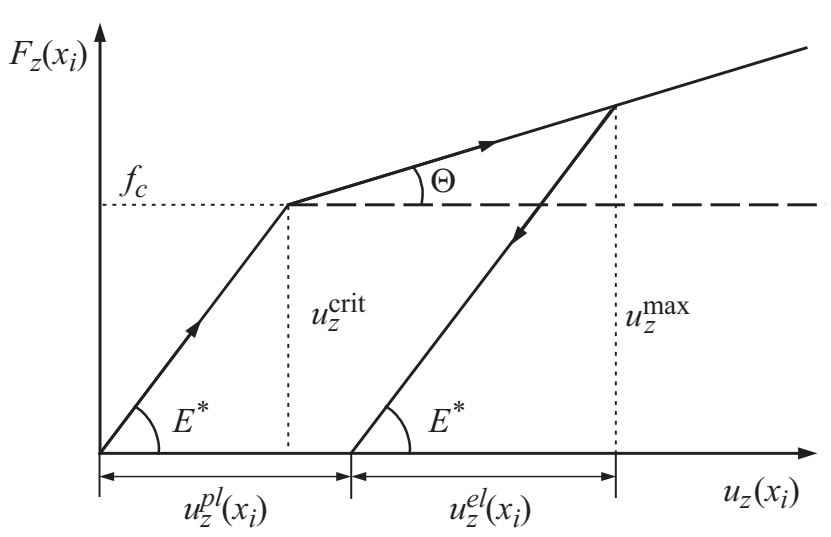

Рис. 1. Схематический вид кривой нагружения для выделенной пружины с координатой $x_{i}$ с деформационным упрочнением материала.

то величина упругой силы задается соотношением

$$
F_{z}\left(x_{i}\right)=f_{c}+\left[u_{z}\left(x_{i}\right)-u_{z}^{\text {crit }}\right] \Theta \Delta x .
$$

Разгружение пружины, которая пластически деформирована, происходит также по гуковскому участку (см. рис. 1), при этом пластическая составляющая смещения пружины может быть найдена как

$$
u_{z}^{p l}\left(x_{i}\right)=\left(u_{z}^{\max }-u_{z}^{\text {crit }}\right)\left(1-\frac{\Theta}{E^{*}}\right) .
$$

В процессе разгружения пружины нормальную силу следует вычислять по формуле (10), где $u_{z}^{p l}\left(x_{i}\right)$ определяется согласно (13). Отметим, что схема, представленная на рис. 1, не единственный способ описания перехода упругой деформации в пластическую. Например, в работе [15] нами использовалась функция, которая описывает плавный переход упругой деформации в пластическую, без разрыва первой производной $\partial F_{z}\left(x_{i}\right) / \partial u_{z}\left(x_{i}\right)$ в точке упруго-пластического перехода. Однако использование такой функции оправдано скорее в случае, когда процесс деформирования исследуется аналитически, а мы строим численную модель. Поэтому мы остановились на более простом варианте, который также позволяет описать деформационное упрочнение.

Процесс нормального столкновения сферического тела с полупространством можно условно разбить на две фазы. Первая фаза - это погружение (индентирование) шарика в полупространство. На протяжении этой фазы нормальная скорость движения шарика будет уменьшаться до нулевого значения, а его кинетическая энергия переходить в упругую энергию сжатых пружин, а также частично затрачиваться на работу пластической деформации. Вторая фаза - это отскок шарика. В этой фазе скорость шарика будет увеличиваться от нуля до значения, с которым он окончательно отделится от полупространства. Поскольку в обоих фазах шарик всегда движется в противоположных направлениях, для каждой пружины будет сначала осуществляться нагружение с монотонно увеличивающимся значением $u_{z}\left(x_{i}\right)$, а при разгружении величина $u_{z}\left(x_{i}\right)$ будет монотонно уменьшаться.

Теперь рассмотрим процесс столкновения сферы с полупространством. Пускай начальная скорость шарика равна $v_{z 0}$. Рассмотрим ситуацию, в которой шарик не вращается (начальная циклическая частота равна нулю). При этом он при отлете от поверхности также будет иметь нулевую циклическую частоту вращения. Одной из основных величин в таких экспериментах является коэффициент восстановления нормальной скорости $e_{n}$, вычисляемый как

$$
e_{n}=-\frac{v_{z}}{v_{z 0}}
$$

где $v_{z}-$ скорость шарика, с которой он отлетает от поверхности полупространства (скорости $v_{z}$ и $v_{z 0}$ разнонаправленны и имеют противоположные знаки, поэтому $\left.e_{n}>0\right)$. Для определения временной эволюции скорости шарика $v_{z}$ необходимо решить единственное уравнение движения (поскольку отсутствует тангенциальное движение), записанное в виде

$$
m \dot{v}_{z}=-F_{z}
$$

где нормальная сила $F_{z}$ при контакте шарика с поверхностью согласно выражению (6) всегда положительна. Решая уравнение (15) с использованием описанной выше процедуры, можно найти конечную скорость шарика (при его отлете от поверхности полупространства), а значит и величину коэффициента восстановления $e_{n}(14)$.

\section{3. Результаты моделирования нормального соударения и сравнение с экспериментом}

В работе [16] экспериментально определялась зависимость коэффициента восстановления нормальной скорости $e_{n}(14)$ от значения начальной нормальной скорости $v_{z 0}$. А именно, изучалось отражение шарика от гладкой стальной пластины размером $25.4 \times 22.86 \mathrm{~cm}$ и толщиной $5.08 \mathrm{~cm}$. Диаметр шарика составлял $1.27 \mathrm{~cm}$. В работе [16] указывается, что коэффициент восстановления сильно зависит от качества обработки контактирующих поверхностей. Это вполне логично, поскольку в случае, когда поверхности контактирующих тел имеют шероховатости, на этих шероховатостях при соударении будут создаваться существенно большие напряжения, чем в объеме шарика. Это приведет к деформации неровностей и диссипации механической энергии. Более того, всегда при ударе слышен звук, являющийся следствием распространения упругих волн в материале. А это также связано с диссипацией энергии. Дополнительно стоит учитывать, что эксперименты часто проводят в воздухе, который также оказывает вязкое сопротивление. За счет этих факторов, коэффициент восстановления 


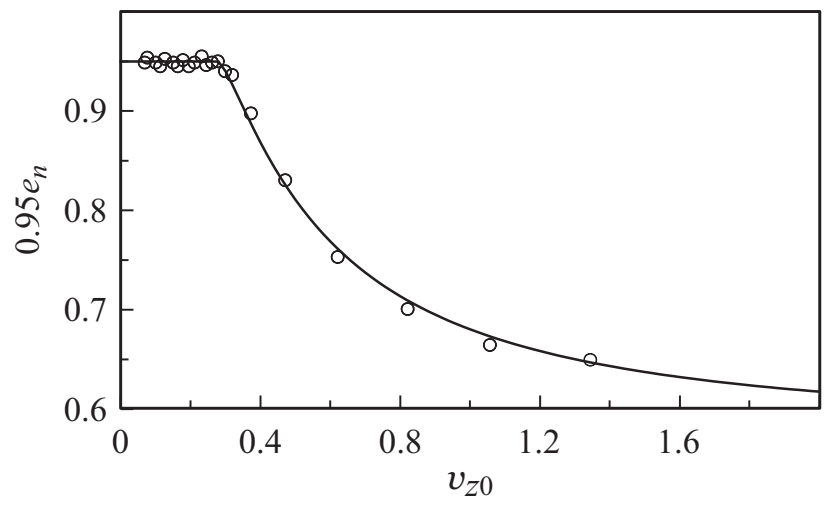

Рис. 2. Коэффициент восстановления нормальной скорости $e_{n}(14)$ как функция начальной скорости шарика $v_{z 0}$. Сплошная кривая показывает результаты расчета в рамках метода MDR, символы - экспериментальные результаты из работы [16].

в упругой области соударения в работе [16] при чисто обработанных поверхностях контактирующих тел не превышал 0.82. После дополнительной обработки поверхностей мелкозернистой наждачной бумагой коэффициент восстановления стал достигать величины 0.87 . И наконец, после тщательной полировки с применением ультрадисперсного абразива, коэффициент восстановления стал достигать максимальной величины $e_{n} \approx 0.95$.

Поскольку мы рассматриваем случай столкновения сферического тела с пластиной, когда они изготовлены из одинаковых материалов, формула (3) примет более простой вид

$$
E^{*}=\frac{E}{2\left(1-v^{2}\right)},
$$

что для параметров стали $E=2 \cdot 10^{11} \mathrm{~Pa}$ и $v=0.286$ [16] даст значение $E^{*} \approx 1.09 \cdot 10^{11} \mathrm{~Pa}$. Радиус шарика составлял $R=0.635 \mathrm{~cm}$, плотность стали $\rho=7.8 \mathrm{~g} / \mathrm{cm}^{3}$, соответственно его масса $m=4 \pi \rho R^{3} / 3 \approx 8.366 \mathrm{~g}$.

Предлагаемая нами модель упруго-пластического столкновения предполагает идеально гладкие поверхности контактирующих тел, и в этом случае при малых скоростях нормального соударения (когда реализуется чисто упругое столкновение) коэффициент восстановления $e_{n}=1$. Однако, как мы это указали выше, за счет влияния факторов, которые не учитываются в нашей модели, в работе [16] максимальное значение коэффициента восстановления нормальной скорости $e_{n}$ не превышало значение 0.95 . Поэтому при дальнейших расчетах мы производили нормировку коэффициента восстановления на это максимальное значение.

На рис. 2 показана зависимость рассчитанного значения коэффициента восстановления при параметрах, соответствующих эксперименту, описанному в работе [16] (эти параметры указаны выше).

Используемое в модели значение коэффициента упрочнения при этом составляло $\Theta=0.37 E^{*}$, а критическая плотность силы $q_{c}=3.8 \cdot 10^{5} \mathrm{~N} / \mathrm{m}$. Эти два параметра в работе [16] не указаны, поэтому они подбирались таким образом, чтобы теоретически получаемая кривая, показанная на рис. 2, максимально соответствовала экспериментальным данным. При этом параметр $q_{c}$ на зависимости $e_{n}\left(v_{z 0}\right)$ задает точку, в которой начинается пластическая деформация (т.е. величина $e_{n}$ начинает убывать), а значение параметра $\Theta$ задает вид кривой $e_{n}\left(v_{z 0}\right)$ в области наличия пластических деформаций. Как видно из рисунка, предложенная нами модель хорошо описывает конкретную экспериментальную ситуацию.

Существуют работы, в которых экспериментально исследуются косые столкновения, и измеряется тангенциальный коэффициент восстановления, а также изменение циклической частоты вращения шарика после удара, например работа [17]. Причем в [17] при тангенциальном движении необходимо учитывать трение, которое будет приводить к частичному проскальзыванию в зоне контакта. В рамках метода MDR нами была построена такая существенно более сложная численная модель, однако не удалось подобрать соответствующие параметры для удовлетворительного описания экспериментальных результатов. Это говорит о том, что в работе [17] реализовался другой механизм пластической деформации. Более того, в [17], как частный случай отдельно исследуется нормальное соударение, и полученная зависимость $e_{n}\left(v_{z 0}\right)$ существенно отлична от показанной на рис. 2. Не удалось нам с помощью предлагаемого механизма пластичности описать и результаты работы [18] (см. также [19]), в которой также изучается нормальное столкновение частиц. Более того, теория, предложенная в [19], тоже довольно плохо описывает экспериментальные результаты работы [18]. Все это говорит о том, что в реальности пластическая деформация может происходить по многим сценариям, и для каждого необходим свой отдельный подход. Здесь мы предложили один из вариантов описания нелокальной пластичности, справедливость которого подтверждена экспериментальной работой [16].

\section{4. Заключение}

В работе предложена численная модель, описывающая нормальное столкновение сферических тел в упругопластической области. Показано, что с ростом величины начальной нормальной скорости коэффициент восстановления нормальной скорости уменьшается. Было проанализировано несколько экспериментальных работ, содержащих данные по исследованию коэффициента восстановления нормальной скорости и показано, что предложенная нами модель подходит далеко не для всех ситуаций. А именно, мы описываем случай нелокальной пластичности, в котором теряет смысл критическое напряжение текучести материала. Только в этом специфическом случае можно использовать изложенную выше теорию нормального соударения. В связи с этим, 
результаты, полученные в данной работе, могут быть использованы для определения механизма деформирования. Дело в том, что для всех систем существует свой критический радиус индентора, меньше которого предложенный нами подход становится применимым. Если радиус больше этого значения, все изложенное выше будет неверно. В связи с этим такую методику моделирования можно использовать для определения этого критического радиуса.

\section{Список литературы}

[1] M.P. Ciamarra, A.H. Lara, A.T. Lee, D.I. Goldman, I. Vishik, H.L. Swinney. Phys. Rev. Lett. 92, 194301 (2004).

[2] P. Jop, Y. Forterre, O. Pouliquen. Nature 441, 727 (2006).

[3] N.V. Brilliantov, F. Spahn, J.-M. Hertzsch, T. Pöschel. Phys. Rev. E 53, 5382 (1996).

[4] F.G. Bridges, A. Hatzes, D.N.C. Lin. Nature 309, 333 (1984).

[5] J.R. Barber. Appl. Math. Phys. (ZAMP) 30, 468 (1979).

[6] I.A. Lyashenko. FU Mech. Eng. 14, 313 (2016).

[7] В.В. Ефименко. Вестн. ФГОУ ВПО МГАУ 2, 41 (2009).

[8] C. Thornton, Z. Ning. Powder Technol. 99, 154 (1998).

[9] V.L. Popov, M. Heß. Method of dimensionality reduction in contact mechanics and friction. Springer-Verlag, Berlin (2015). 265 p.

[10] I.A. Lyashenko, V.L. Popov. Sci. Rep. 5, 8479 (2015).

[11] E. Willert, V.L. Popov. Z. Angew. Math. Mech. (ZAMM) 96, 1089 (2016).

[12] I.A. Lyashenko, E. Willert, V.L. Popov. Mech. Mater. 92, 155 (2016).

[13] V.L. Popov. FU Mech. Eng. 13, 39 (2015).

[14] А.Д. Погребняк, С.Н. Братушка, В.M. Береснев, N. Levintant-Zayonts. Успехи химии 82, 1135 (2013).

[15] А.В. Хоменко, Я.А. Ляшенко. Трение и износ 31, 412 (2010).

[16] J.M. Lifshitz, H. Kolsky. J. Mech. Phys. Solids 12, 35 (1964).

[17] D.A. Gorham, A.H. Kharaz. Powder Technol. 112, 193 (2000).

[18] H.F. Kagnur, I.R. Kleis. Изв. АН CCCP. MTT 5, 182 (1988).

[19] O.V. Kim, P.F. Dunn. J. Aerosol Sci. 38, 532 (2007). 